\title{
Internal and External Influences on the Rate of Sensory Evidence Accumulation in the Human Brain
}

\author{
Simon P. Kelly ${ }^{1}$ and Redmond G. 0'Connell ${ }^{2}$ \\ ${ }^{1}$ Department of Biomedical Engineering, City College of the City University of New York, New York, New York 10031, and ${ }^{2}$ Trinity College Institute of \\ Neuroscience and School of Psychology, Trinity College Dublin, Dublin 2, Ireland
}

We frequently need to make timely decisions based on sensory evidence that is weak, ambiguous, or noisy resulting from conditions in the external environment (e.g., a cluttered visual scene) or within the brain itself (e.g., inattention, neural noise). Here we examine how externally and internally driven variations in the quality of sensory evidence affect the build-to-threshold dynamics of a supramodal "decision variable" signal and, hence, the timing and accuracy of decision reports in humans. Observers performed a continuousmonitoring version of the prototypical two-alternative dot-motion discrimination task, which is known to strongly benefit from sequential sampling and temporal accumulation of evidence. A centroparietal positive potential (CPP), which we previously established as a supramodal decision signal based on its invariance to motor or sensory parameters, exhibited two key identifying properties associated with the "decision variable" long described in sequential sampling models: (1) its buildup rate systematically scaled with sensory evidence strength across four levels of motion coherence, consistent with temporal integration; and (2) its amplitude reached a stereotyped level at the moment of perceptual report executions, consistent with a boundary-crossing stopping criterion. The buildup rate of the CPP also strongly predicted reaction time within coherence levels (i.e., independent of physical evidence strength), and this endogenous variation was linked with attentional fluctuations indexed by the level of parieto-occipital $\alpha$-band activity preceding target onset. In tandem with the CPP, build-to-threshold dynamics were also observed in an effector-selective motor preparation signal; however, the buildup of this motor-specific process significantly lagged that of the supramodal process.

\section{Introduction}

According to sequential sampling models, accurate perceptual decisions are achieved in the face of sensory noise by integrating evidence over time into a "decision variable" that triggers an action upon reaching a criterion (Smith and Vickers, 1988; Smith and Ratcliff, 2004). Primate neurophysiology studies have established that such a quantity is explicitly encoded in the firing rate of neurons in frontal (Kim and Shadlen, 1999) and parietal (Shadlen and Newsome, 2001) oculomotor areas. Two cardinal properties identify these neuronal signals with the theoretical decision variable: (1) they reach a stereotyped level on action execution, indicating a boundary-crossing effect; and (2) they build over time at a rate that scales with the strength of the sensory evidence, consistent with temporal integration (Gold and Shadlen, 2007). Although a concerted effort has been made to isolate analogous signals in the human brain (Heekeren et al., 2008), no study has yet identified a signal whose buildup rate

Received Aug. 6, 2013; revised 0ct. 2, 2013; accepted Nov. 3, 2013.

Author contributions: S.P.K. and R.G.O. designed research; S.P.K. and R.G.O. performed research; S.P.K. and R.G.O. analyzed data; S.P.K. and R.G.O. wrote the paper.

This work was supported by the Irish Research Council New Foundations Scheme. We thank Eimear Hayes and Deirdre Twomey for their assistance with data collection, Lucas Parra for advising on the single-trial classification approach, and Peter Murphy and Clare Kelly for their helpful comments during the preparation of the manuscript. The authors declare no competing financial interests.

Correspondence should be addressed to Dr. Redmond G. O'Connell, Trinity College Institute of Neuroscience and School of Psychology, Trinity College Dublin, Dublin 2, Ireland. E-mail: reoconne@tcd.ie.

DOI:10.1523/JNEUROSCI.3355-13.2013

Copyright $\odot 2013$ the authors $\quad 0270-6474 / 13 / 3319434-08 \$ 15.00 / 0$ deterministically mediates the relationship between evidence strength and reaction time (RT).

Recently, we identified a decision signal (centroparietal positivity [CPP]) in the human EEG that exhibited a boundary-crossing relationship with perceptual reports when participants detected gradual intensity changes in a continuously presented stimulus (O'Connell et al., 2012). Further, we showed that this signal is supramodal: it builds during decision formation regardless of sensory parameters or motor requirements. However, because the sensory evidence itself increased over time at a rate that was fixed across trials, it was not possible to establish the dependence of buildup rate on evidence strength. Although a monotonic relationship has been well established in premotor decision signals in monkeys, it is uncertain whether a distinct supramodal counterpart in humans should exhibit the same relationship, especially given that CPP onset latency appeared to be a far stronger determinant of RT than buildup rate for the fixed contrast-change targets of our previous study (O'Connell et al., 2012). Here, we addressed this question using a continuousmonitoring version of the prototypical random dot motion (RDM) (Newsome et al., 1989, Britten et al., 1992) direction-discrimination task, in which a continuously playing dot-motion patch steps from zero to one of four non-zero, constant coherence levels at intermittent times. Seamless coherence transitions avoid sudden intensity changes, which would otherwise elicit large sensory-evoked potentials that mask simultaneously active decision processes due to global signal summation.

Our paradigm additionally allowed us to examine the relative timing of evidence accumulation dynamics in supramodal versus 
effector-selective decision variable signals. Which process should evolve first is uncertain because several signals resembling the CPP are thought to peak after the first-order decision (e.g., Nieuwenhuis et al., 2005; Murphy et al., 2012). Finally, we tested the extent to which across-trial variability in CPP buildup rate predicts RT within coherence levels (i.e., independently of the physical evidence), and further, the extent to which buildup rate itself varies with fluctuations of attentional engagement reflected in pretarget levels of posterior $\alpha$-band activity (e.g., O'Connell et al., 2009).

\section{Materials and Methods}

Participants. Eighteen participants gave written informed consent, and all procedures were approved by the ethical review board of the School of Psychology, Trinity College Dublin. Ethical guidelines were in accordance with the Declaration of Helsinki. All participants were between 21 and 35 years of age, had normal or corrected-to-normal vision, and had no history of psychiatric diagnosis, sensitivity to flickering light, or head injury. To ensure sufficient trials for all analyses, participants were only included if, after artifact rejection and exclusion of incorrect and miss trials, they had at least 15 trials available for each of the four coherence levels in each of the two motion directions. This led to the exclusion of 5 participants leaving a final total of 13 (6 female).

Continuous RDM task. We used a continuous version of the classic RDM task (Newsome et al., 1989; Britten et al., 1992) in which participants monitored a patch of incoherently moving dots for intermittent targets defined by $1.9 \mathrm{~s}$ periods of coherent motion in the leftward or rightward direction. In contrast with the typical discrete-trial RDM task in which moving dots appear suddenly, our targets were defined by a seamless step transition from incoherent to coherent motion, thus eliminating transient sensory-evoked potentials at evidence onset (see Fig. $1 A$ ). Motion direction and coherence level varied independently and randomly on a target-by-target basis. Coherence could take one of four values: $25 \%, 35 \%, 50 \%$, or $70 \%$. To facilitate measurement of a motor preparatory signal of evidence accumulation in tandem with the supramodal CPP, we asked subjects to indicate leftward motion with a lefthand button press and rightward motion with a right-hand button press (for a similar approach, see Donner et al., 2009; de Lange et al., 2013). Participants were instructed to respond as soon as they were sure of the motion direction. The intertarget interval, during which incoherent motion was continuously displayed, lasted $3.6,6.6$, or $8.4 \mathrm{~s}$, chosen randomly on a trial-by-trial basis. A total of 48 targets (6 of each direction-coherence combination) were presented within each block of just $<7$ min.

Data recording and task performance took place in a dark soundattenuated room with participants seated at a distance of $75 \mathrm{~cm}$ from the visual display. Visual stimuli were presented against a dark gray background on a $51 \mathrm{~cm}$ CRT monitor operating at a refresh rate of $85 \mathrm{~Hz}$ and resolution of $1280 \times 960$. Participants were instructed to fixate on a centrally presented $5 \times 5$ pixel white square at all times during task performance. The RDM stimulus was presented within a 5 degree aperture centered on fixation. During incoherent motion, an average of 118 white dots (each $4 \times 4$ pixels) were placed randomly and independently within the circular aperture on each of a sequence of $26 \mathrm{~ms}$ frames played at 14.17 frames/s. This resulted in an on-off flicker at the same rate, giving rise to a steady-state visual-evoked potential (SSVEP) in the EEG. During coherent motion, a proportion of the dots were randomly selected on each frame to be displaced by a fixed distance of 0.24 degrees in either the left or right direction on the following frame, resulting in a motion speed of $3.33 \mathrm{deg} / \mathrm{s}$. It should be noted that SSVEP amplitude encodes the intensity of the stimulus rather than motion coherence and therefore does not index the encoding of sensory evidence in the RDM task as it did in the contrast change-detection tasks of our previous study.

Participants completed two short practice sessions before data recording. In the first practice, all targets were presented at a coherence of $80 \%$ to ensure that each participant understood the nature of the task. Participants performed the task until they were able to correctly identify 5 consecutive targets. In a second practice session, the four coherence levels were introduced and the participant performed the task for 3 min (25 target trials) during which time verbal feedback was provided on hits, misses, and false alarms. Participants were given a rest break of $\sim 30 \mathrm{~s}$ duration after each testing block. Each participant completed 7-10 blocks of the task (mean \pm SD, $9.6 \pm 0.8$ ).

Behavioral data analysis. Behavioral performance was analyzed by conducting a repeated-measures ANOVA with factors of motion direction (left vs right) and coherence $(25 \%, 35 \%, 50 \%, 70 \%)$ on miss rate, RT, and incorrect responses. We additionally fitted RT data to a drift diffusion model to verify that, consistent with previous work (e.g., Ratcliff and McKoon, 2008), the behavioral data are well explained by a model in which only drift rate varies as a function of coherence. For this, we used a drift-diffusion model-fitting procedure implemented in the DMA toolbox (Vandekerckhove and Tuerlinckx, 2008), first with only the drift rate parameter allowed to vary across coherence, second with only boundary separation allowed to vary, and third with both drift rate and boundary separation allowed to vary, to test the relative goodness-of-fit for these three models (Bayes Information Criterion [BIC]). The other fitted parameters that were constrained to be equal across coherence levels were the mean and range of nondecision time (the duration of all processes apart from the decision process), intertrial range in starting-point and across-trial drift-rate variability. Starting point (response bias) was fixed at halfway between decision boundaries (neutral) in all model fits. Because the differences in BIC values had a clearly non-Gaussian distribution across subjects, two Wilcoxon signed-rank tests were used to test whether the first model allowing only drift rate to vary with coherence achieved a lower median BIC (better fit) than either of the other two models.

EEG acquisition and preprocessing. Continuous EEG was acquired using an ActiveTwo system (BioSemi) from 128 scalp electrodes, digitized at $512 \mathrm{~Hz}$. Vertical and horizontal eye movements were recorded using two vertical electro-oculogram (EOG) electrodes placed above and below the left eye and two horizontal EOG electrodes placed at the outer canthus of each eye, respectively. Data were analyzed using custom scripts in MATLAB (MathWorks), drawing on EEGLAB routines for reading data files and spherical spline interpolation of noisy channels (Delorme and Makeig, 2004). No high-pass filter was applied either online or offline. EEG data were rereferenced offline to the average reference and low-pass filtered up to a $45 \mathrm{~Hz}$ cutoff using a two-way leastsquares FIR filter. Target epochs were extracted using a window of -0.75 $\mathrm{s}$ to $2 \mathrm{~s}$ around target onset. Trials were rejected if the bipolar vertical EOG signal (upper minus lower) exceeded an absolute value of $200 \mu \mathrm{V}$ or if any scalp channel exceeded $100 \mu \mathrm{V}$ at any time during the interval extending from the start of the epoch to $200 \mathrm{~ms}$ after response execution. The resulting data were converted to current source density (CSD) (Kayser and Tenke, 2006) to increase spatial selectivity and minimize volume conduction. This step was introduced to minimize the projection of motor preparation signals at frontocentral electrodes to the posterior CPP electrodes (see Impact of CSD transformation).

Measurement of supramodal and effector-specific decision signals. CPP waveforms were generated for each participant by averaging the singletrial epochs, which were baseline-corrected relative to the $200 \mathrm{~ms}$ interval ending at target onset. CPP amplitude and latency were measured from electrodes centered on the region of maximum component amplitude identified in the grand average scalp topography (2 electrodes closest to standard site CPz, consistent with O'Connell et al., 2012). Responselocked ERPs were derived by extracting epochs from -1000 to $100 \mathrm{~ms}$ relative to the time of button press on each trial, retaining the same prestimulus baseline as the stimulus-locked waveforms. The lateralized readiness potential (LRP), an index of unimanual motor preparation (Gratton et al., 1988; de Jong et al., 1988; Eimer, 1998), was measured by subtracting ipsilateral from contralateral ERPs at a pair of frontocentral electrodes close to standard sites FC3 and FC4 and averaging across motion direction. These electrodes were selected based on inspection of the grand-average difference topography for leftward minus rightward motion targets at response execution (see Fig. 1D).

Impact of coherence on signal buildup rate. The impact of coherence on signal buildup rate was examined for both the CPP and LRP, testing both the stimulus-aligned and response-aligned waveforms in each case. 
Buildup rate was measured as the slope of a straight line fitted to the unfiltered ERP waveform of each subject, using the interval 200 to $350 \mathrm{~ms}$ for the stimulus-aligned CPP, 300 to $450 \mathrm{~ms}$ for the stimulus-aligned LRP, -250 to $-100 \mathrm{~ms}$ for the response-aligned CPP, and -300 to -150 ms for the response-aligned LRP (see Fig. 1).

Relative timing of supramodal and effector-specific decision signals. To explore the temporal relationship between the CPP and LRP, we measured the peak amplitude of the two signals in a $100 \mathrm{~ms}$ window centered on response execution for each subject and identified the first preceding time-point in the response-aligned average at which the signals reached half of this value (see Fig. 1C,D). To establish the time intervals over which evidence strength impacted on the buildup rate of the CPP and LRP signals, we measured the temporal slope of each signal in each subject's average waveform in a moving window of $100 \mathrm{~ms}$ covering the interval from stimulus onset to response execution in $10 \mathrm{~ms}$ steps. Again, buildup rate was computed as the slope of a straight line fitted to the unfiltered signal within each temporal window. Signal buildup rate was then regressed against coherence for each time window and those for which the regression slope significantly differed from zero across all subjects in the direction expected for each signal (positive regression slopes for the positive-going CPP, negative for the negative-going LRP, one tailed tests with $p<0.05$ ) are marked below the waveforms in Figure $1 C, D$.

Influence of attentional fluctuations on decision signal buildup rate. To examine the relationship between RT and CPP buildup rate independently of coherence, and link such correlated variation to pretarget EEG indices of attentional engagement ( $\alpha$ band activity), we conducted two analyses. To simply test for any within-subjects relationship between RT and CPP buildup rate and/or pretarget $\alpha$, we divided the trials of each coherence level of each subject into two RT bins based on a median split and conducted a $2 \times 4$ ANOVA with the factors of RT bin and coherence, for each of the dependent variables of CPP buildup rate and pretarget $\alpha$.

Next, to establish the more fine-grained variation of CPP buildup rate and/or pretarget $\alpha$ as a function of RT, we conducted a set of analyses in which all trials of all participants were pooled to facilitate finer trial binning. For each single trial, we computed CPP buildup rate using the same linear fitting method and stimulus-aligned and response-aligned intervals as above and calculated pretarget $\alpha$ band amplitude via the standard FFT of a pretarget window of $777 \mathrm{~ms}$ (fitting exactly 9 cycles of the SSVEP to minimize spectral leakage). We integrated spectral amplitude over the $\alpha$ frequency range of $8-13 \mathrm{~Hz}$ (more precisely $7.7-12.9 \mathrm{~Hz}$ given window size) and $>10$ parieto-occipital electrodes covering the area from standard 10-20 site PO7, through POz, to PO8. Before trial sorting, we standardized ( $z$-score transformed) single-trial RTs within each subject regardless of coherence to guard against the possibility that relationships with RT could arise because of differences across, rather than within, subjects. Trials were then divided into 6 RT bins within each coherence level.

To examine the variation of CPP buildup with RT, we plotted the average $\mathrm{CPP}$ within each of the $6 \mathrm{RT}$ bins, collapsing across coherence in each bin (see Fig. $2 A$, middle and right). To enable statistical testing, we captured the relationship between RT and CPP buildup rate within coherence levels as the slope of a linear fit of CPP buildup rate against RT bin. We performed a permutation test to quantify the probability that the pattern we observed could arise by chance given the null hypothesis of no relationship between CPP buildup rate and RT within coherence level. A permutation test furnishes a null distribution directly from the data by permuting the data in accordance with this null hypothesis. In the present case, we randomly permuted the assignment of RT to trials within each coherence level of each subject, directly simulating the scenario that, regardless of whether there is a coherence effect and/or individual differences, the CPP buildup rate on single trials within each coherence and subject has no relationship to RT or $\alpha$. This randomization was repeated 10,000 times, and the actual observed effect was then located as a percentile on the thus-formed null distribution to quantify the probability that it arose by chance.

To examine the variation of pretarget $\alpha$ with RT, we repeated the above steps for plotting and permutation testing, this time substituting the CPP buildup rate for our single-trial pretarget $\alpha$ measures (see Fig. $2 A$, left).

Even if sorting by RT produces significant relationships with both pretarget $\alpha$ and CPP buildup rate, it is possible that they have independent but additive effects on RT and may themselves not be related. To rule this out, we again sorted trials into 6 bins for each coherence level, but this time sorting by $\mathrm{CPP}$ buildup rate rather than RT. Given that $\mathrm{CPP}$ buildup rate was used as the sorting variable in this analysis, it was critical to maximize the robustness of single-trial measurements. To this end, we used a single-trial classifier approach developed by Parra et al. (2005). In this procedure, a channel-weight vector " $w$ " (i.e., a "direction" in the 128-channel data) is derived in the multivariate ERP data, which maximizes the accuracy of classification between two training sets, in this case, activity at the time of decision commitment versus during the intertarget interval. Importantly, we used data samples in the intertrial interval that were baseline-corrected in the same way as the samples extracted from decision commitment time (i.e., using a baseline interval a comparable amount of time before the training samples), so that spurious slow drift in some electrodes could not skew the discriminating direction in the data. Specifically, whereas the "decision-formation" training samples were extracted from unfiltered signals in the window -180 to $-80 \mathrm{~ms}$ before response execution, baseline-corrected relative to the $200 \mathrm{~ms}$ pretarget window as usual, the "no-decision" training samples were extracted from the window -100 to 0 relative to target onset, baselinecorrected relative to the interval -750 to $-650 \mathrm{~ms}$. The discriminating vector "w" was derived independent of coherence, and separately for each individual subject, and was then applied to extract a single decision signal component from both the stimulus-aligned and response-aligned 128-channel single-trial data before pooling. Subject-wise $z$-scoring, pooling, sorting, and binning by CPP buildup rate then proceeded in the same way as for the RT binning procedure, with the exception that outlying values of CPP buildup rate were avoided by trimming the top and bottom $2.5 \%$ of trials. A permutation test was again used to quantify the probability that the observed pattern arose by chance, this time with 10,000 random reassignments of CPP buildup rate to trials within each coherence level of each subject.

\section{Results}

\section{Behavior}

Thirteen subjects performed a continuous version of the RDM task in which $1.9 \mathrm{~s}$ periods of coherent motion at $25 \%, 35 \%, 50 \%$, or $70 \%$ occurred intermittently within a continuous stream of otherwise incoherent motion (Fig. $1 A$ ). In contrast with discrete, forced-choice versions of this task (e.g., Ratcliff and McKoon, 2008), errors on our continuous version arise far more often from missing the temporally unpredictable, seamless transition to coherent motion and failing to respond by the end of the coherent motion interval than from incorrect choices on detected targets $(<1.5 \%$ of trials at any single coherence level, with no main effect of coherence). Increasing the strength of sensory evidence resulted in fewer misses (Fig. $1 B ; F_{(3,36)}=42.1, p<0.001$ ) and faster RTs (Fig. $1 C ; F_{(3,36)}=149.6, p<0.001$ ) with no main effect of motion direction or motion direction by coherence interaction in either case.

Fitting individual behavioral data to a drift-diffusion model with only the drift rate parameter allowed to vary across coherence levels (using the DMA toolbox of Vandekerckhove and Tuerlinckx, 2008) resulted in the expected increase of drift rate with coherence. A within-subjects comparison of goodness-of-fit (BIC) revealed that this model provided a significantly better fit across subjects than a model where only the decision boundary separation was allowed to vary across coherences (Wilcoxon signed rank test for zero median of the BIC difference, $p<$ 0.0005). Because the BIC incorporates a penalty term for the number of free parameters, the model fit with freely varying drift rate also produced a better average BIC than a model in which 
A
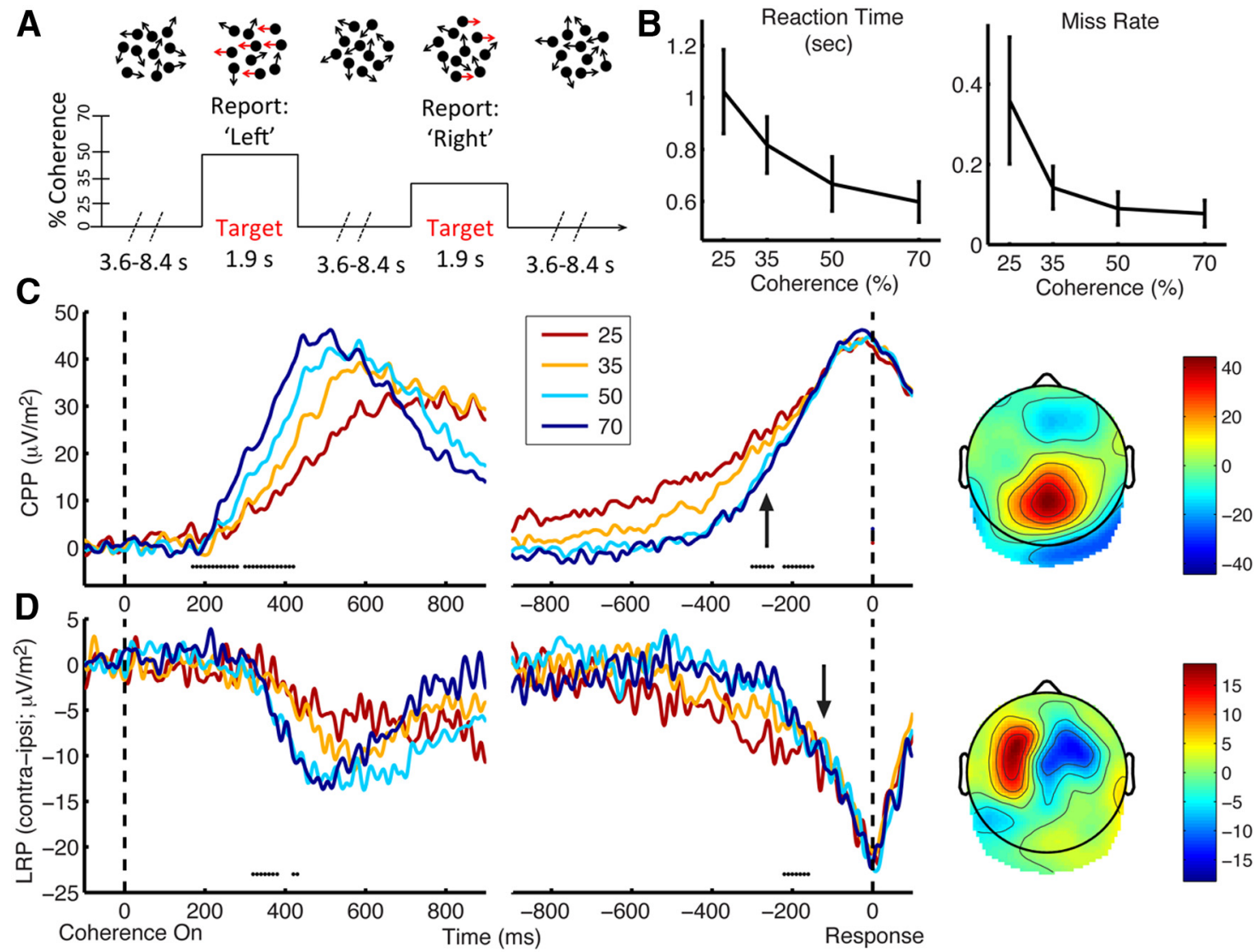

Figure 1. Integration-to-threshold dynamics during motion discrimination in the human brain. $\boldsymbol{A}$, Schematic of the continuous RDM task. Participants monitored a centrally presented dot kinetogram for step transitions from random to coherent motion. $\boldsymbol{B}, \mathrm{RT}$ (left) and miss rate (right) decreased as a function of coherence across the 13 subjects. Error bars indicate SEM. C, CPP waveforms aligned to stimulus onset (left) and response execution (middle) and signal scalp topography (right, color bar represents signal amplitude). D, LRP waveforms aligned to stimulus onset (left) and response execution (middle) measured as the contralateral minus ipsilateral potential difference over frontocentral sites, and the scalp topography of the difference between left motion and right motion trials at the time of response execution (right). Both signals exhibit a gradual buildup whose rate is proportional to the strength of coherent motion and which terminates at a stereotyped potential. Markers running along the bottom of plot $\boldsymbol{C}$ and $\boldsymbol{D}$ indicate the center of $100 \mathrm{~ms}$ time windows in which a linear contrast of signal slope as a function of coherence reached significance (one-tailed based on prediction of faster signal buildup with increasing coherence, $p<0.05$ ), and arrows indicate the point at which each signal reaches half of its peak voltage (averaging across coherences), highlighting that the evidence-dependent buildup of the supramodal CPP precedes that of the effector-selective LRP.

both drift rate and boundary separation were free to vary across coherence levels $(p<0.0005)$.

\section{Impact of coherence on neural evidence accumulation}

Coherent motion elicited the same rising positivity over centroparietal scalp as was observed for gradual stimulus feature changes in our previous study (O'Connell et al., 2012) (Fig. 1C). The CPP topography, amplitude, onset latency, and peak latency did not differ for leftward and rightward motion (all $p>0.05$ ); therefore, waveforms are collapsed across direction throughout. As intended, the seamless transitions from incoherent to coherent motion eliminated sensory-evoked deflections from the centroparietal ERP allowing the evolution of the CPP to be traced from its onset to its peak. Although coherence discontinuously stepped from zero to a constant non-zero level, the CPP exhibited a gradual buildup that reached a stereotyped potential at the time of commitment (Fig. 1C). In accordance with the integration principle of sequential sampling models, the buildup rate of the CPP increased in proportion with the strength of coherent motion. This effect was significant not only in the stimulus-aligned average $\left(F_{(3,36)}=6.7, p<0.01\right)$ but also in the response-aligned average $\left(F_{(3,36)}=4.3, p<0.05\right)$, which, unlike the stimulusaligned trace, is unaffected by differences in RT distributions across the four coherence levels (Fig. 1C).
Motor preparation lags supramodal evidence accumulation

The preparatory activity of contralateral premotor regions was measured via the LRP. The LRP is observed in the interval preceding voluntary hand movements and arises from the lateralization of ERP activity during activation of a unimanual action (Gratton et al., 1988; de Jong et al., 1988; Eimer, 1998). Although the LRP exhibited the same build-to-threshold dynamics as the CPP and a similar dependence of buildup rate on evidence strength in both stimulus $\left(F_{(3,36)}=5.0, p<0.01\right)$ and responsealigned $\left(F_{(3,36)}=4.0, p<0.05\right)$ averages, it lagged the CPP in terms of both the time at which the buildup reached half of its peak (by $113 \pm 87 \mathrm{~ms} \mathrm{t}_{(12)}=4.6, p<0.001$ ) and the time at which a linear regression of signal buildup rate onto coherence in contiguous $100 \mathrm{~ms}$ windows spanning the poststimulus epoch, first reached statistical significance ( $170 \mathrm{~ms}$ for the CPP vs $320 \mathrm{~ms}$ for the LRP; Fig. 1D). To verify that the temporal differences between the two signals were not the result of increased noise in the LRP, we also measured the CPP at two symmetric pairs of electrodes that were anterior and posterior to its centroparietal maximum to artificially reduce the signal-to-noise ratio of the CPP to below that of the LRP. Again, we found that the CPP reached half of its peak voltage significantly earlier than the LRP (by $78 \pm 101 \mathrm{~ms}, t_{(12)}=2.8, p<0.05$ ). 

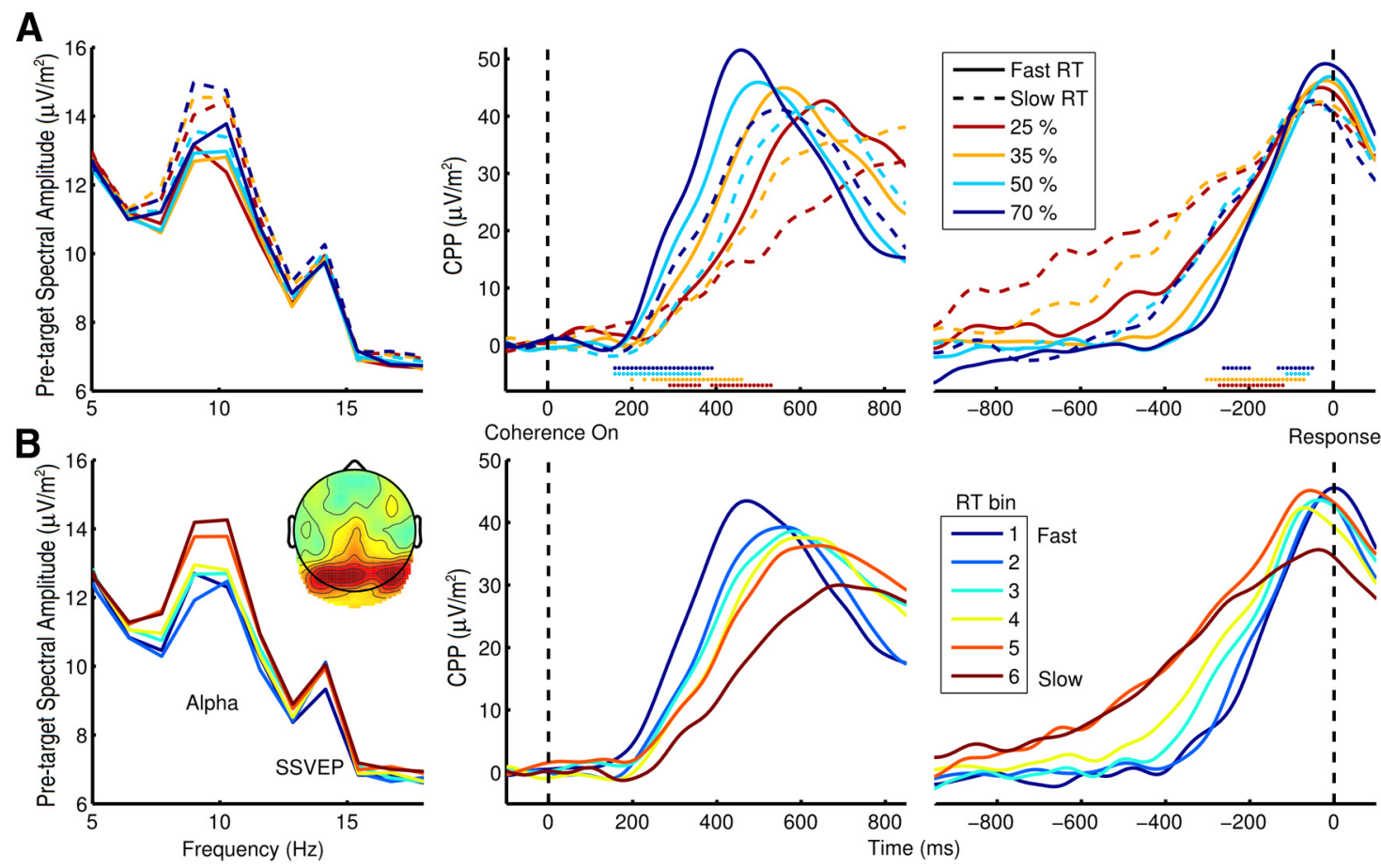

Figure 2. Internal influences on decision speed. $A$, For each participant, single trials within each coherence level were sorted as a function of RT and divided into equal-sized fast and slow bins based on a median split. Faster RTs were preceded by decreased activity in the $8-13 \mathrm{~Hz} \alpha$ band over parieto-occipital electrodes (left). (PP waveforms aligned to stimulus onset (middle) and response (right) show that faster buildup rates led to faster RTs. Markers running along the bottom of each plot indicate the center of 100 ms time windows in which signal buildup rate significantly differed as a function of RT (one-tailed based on prediction of faster RTs with increasing buildup rate, $p<0.05$ ). $\boldsymbol{B}$, Within each coherence level, single trials were pooled across subjects and sorted as a function of RT and divided into six equal-sized bins. Trials in the corresponding bins were then averaged across coherence levels for the plots in this figure. In keeping with the results of the within-subject analysis in $A$, faster RTs were preceded by decreased activity in the $8-13 \mathrm{~Hz} \alpha$ band over parieto-occipital electrodes (see topography inset). Again, RT clearly decreases with CPP buildup rate.

\section{Internal influences on accumulation rate}

To examine endogenous determinants of decision RT, we conducted a within-subjects analysis in which each participant's trials were sorted into equal-sized fast and slow RT bins (based on a median split) at each coherence level and separately averaged. This analysis revealed that steeper buildup of the CPP was associated with significantly faster RT within a given coherence level (stimulus-locked, $F_{(3,36)}=19.3, p<0.001$; response-locked, $F_{(3,36)}=5.6, p<0.05$; Fig. $\left.2 A\right)$. To more finely establish the variation in buildup rate as a function of RT, we conducted an additional single-trial analysis using pooled data from all 13 participants. Pooled trials were sorted within each coherence level according to individually $z$-scored RT and then divided into six equal-sized percentile bins. Figure $2 B$ shows CPP waveforms for all 6 bins, with coherence collapsed within each bin, illustrating a clear increase in buildup rate for trials with decreasing RT. We quantified this effect as the slope of a line fitted to CPP buildup rate against RT bin. A permutation test based on shuffling RT across trials within coherence levels and subjects (see Materials and Methods) confirmed that this effect significantly $(p<0.0001)$ deviated from the null distribution formed by RT shuffling for both the stimulus-locked and response-locked waveforms.

The relatively global nature of EEG confers the advantage of enabling constituent decision processes to be examined in parallel, including processes that play an important supporting role such as attention. Although attentional engagement was not explicitly manipulated in this study, the amplitude of the $\alpha$ rhythm $(8-13 \mathrm{~Hz})$ measured over posterior scalp sites is well known to provide a sensitive index of attention deployed across modalities
(Foxe et al., 1998) and across visual locations (Kelly et al., 2006; Thut et al., 2006), and has been shown to increase significantly over several seconds leading up to an attentional lapse on a continuous detection task (O'Connell et al., 2009). Standard Fourier analysis of the $750 \mathrm{~ms}$ preceding target onset revealed that a similar spectral signature of attentional disengagement preceded targets with longer RTs. Specifically, pretarget $\alpha$-band amplitude increased with increasing RT bin in both the within-subjects (two RT bins per subject as above with $\alpha$ distributions normalized by log-transforming, $F_{(1,12)}=4.95, p<0.05$; Fig. $2 A$, left) and pooled (permutation test as above, $p<0.0001$; Fig. $2 B$, left) analyses. This effect cannot be attributed to a general decrease in arousal or a shift of attention away from the stimulus location because the amplitude of the occipital SSVEP, which was elicited by flickering the dots at a constant rate of $14 \mathrm{~Hz}$, did not decrease systematically as a function of RT (Fig. 2).

A further pooled analysis confirmed that the same relationships between $\alpha$-band power, CPP buildup rate, and RT were preserved when CPP buildup rate was used as the sorting variable (Fig. 3). Within each coherence level, single trials were sorted as a function of CPP buildup rate and divided into six equal-sized bins. Trials in the corresponding bins at each coherence level were then pooled and averaged for this plot. Faster CPP buildup rates were preceded by decreased activity in the $8-13 \mathrm{~Hz} \alpha$ band over parieto-occipital electrodes (permutation test, $p<0.0001$; Fig. $3 A$ ) and followed by faster RTs $(p<0.0001$; Fig. $3 B)$. Ideally, $\alpha$ activity would be used as the sorting variable when relating prestimulus $\alpha$ to CPP buildup rate and RT because it is $\alpha$ that comes first in time. However, in these data, sorting single trials as a function of $\alpha$ activity produced no effect on RT or CPP buildup 

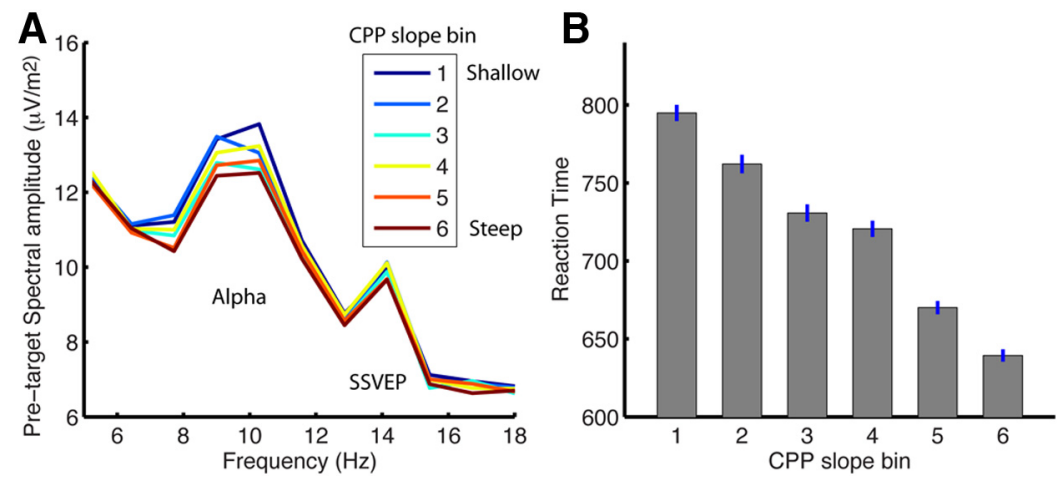

Figure 3. Variability in timing of perceptual reports explained by trial-to-trial changes in decision signal buildup rate. $A$, Faster CPP buildup rates were preceded by decreased activity in the $8-13 \mathrm{~Hz} \alpha$ band over parieto-occipital electrodes. $\boldsymbol{B}$, Faster CPP buildup rates were followed by faster RTs.

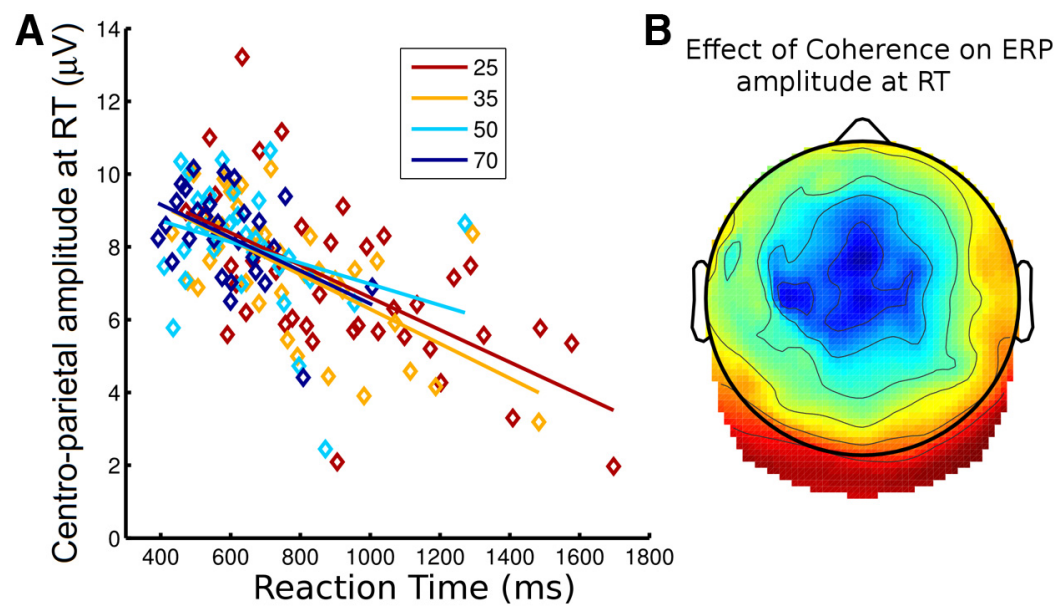

Figure 4. A distinct slow negative potential over frontocentral scalp. $A$, Without applying a CSD transformation, the peak amplitude of the CPP appeared to increase as a function of coherence. This scatter plot of centroparietal amplitude at RT against RT itself indicates that this arises from a trend of decreasing amplitude with RT, not with coherence. Colored lines indicate the linear fit for the relationship between RT and amplitude at each coherence level, which appear colinear. $\boldsymbol{B}$, Topography of the mean difference in CPP amplitude across consecutive pairs of coherence levels, revealing that the decrease in amplitude with RT is driven by a distinct negative-going frontocentral scalp potential, which spatially spreads to centroparietal electrodes in the absence of CSD transformation.

rate. The absence of even an $\mathrm{RT}$ effect, despite the fact that sorting by both RT and CPP buildup rate revealed substantial effects on $\alpha$, indicates that our single-trial measurements of $\alpha$ were not robust on the level that permits capturing attentional fluctuations on a single-trial basis.

\section{Impact of CSD transformation}

Without CSD transformation, the amplitude of the CPP at the time of the decision report apparently decreased as a function of coherence. However, a regression analysis revealed that coherence did not explain a significant amount of variance in CPP amplitude $\left(r^{2}\right.$ change $=0.001, F_{(1,157)}$ change $\left.=0.04, p=0.6\right)$ after accounting for the influence of RT $\left(r^{2}=0.182, F_{(1,158)}=35\right.$, $p<0.001$, based on pooling of single-trial values within each coherence level and dividing into 40 equal-sized bins; Fig. 4A). To investigate the origins of this RT-related effect, the regression of RT against amplitude was repeated at all electrodes and the topography of the resultant $\beta$ weights revealed a distinct negative going frontocentral signal that reached more negative amplitudes at RT for slower trials (Fig. 4B). Together, these results indicate that the differences in CPP amplitude across coherence levels were the result of spatial overlap with this anterior signal, which may correspond to the contingent negative variation or to the bereitschaftspotential, two frontocentral negativities associated with the anticipation of a volitional movement (Brunia and van Boxtel, 2001; Baker et al., 2012). To resolve this issue, we applied CSD transformation, which sharpens topographical resolution by computing a second spatial derivative and thus reduces overlap between distinct topographical foci. Here CSD transformation reduced the influence of the frontocentral negativity on centroparietal electrodes and eliminated the amplitude differences across coherence levels for the CPP, consistent with sequential sampling model predictions and the behavioral modeling results reported above.

\section{Discussion}

We have demonstrated that decisionrelated activity measured in the classically derived human event-related potential over centroparietal scalp exhibits two key defining properties of the theoretical "decision variable" of sequential sampling models: (1) a buildup rate that scales with the strength of sensory evidence and (2) a threshold relationship to perceptual reports. Although numerous studies have identified EEG and MEG signals whose amplitude correlates in some way with decision making performance and/or fitted parameters of sequential sampling models, such as the drift-diffusion model (e.g., Philiastides et al., 2006; Donner et al., 2009; Ratcliff et al., 2009; de Lange et al., 2010; Bode et al., 2012; van Vugt et al., 2012), this is the first study to directly validate the core prediction of evidence strength-dependent temporal integration in a unitary human brain signal. A general implication of these findings is that the CPP provides access to specific parameters of the decision making process (evidence accumulation rate and decision criterion) in the human brain via simple, single-trial signal measurements (slope and amplitude). More specifically, the present results speak to the issues of how endogenous fluctuations combine with exogenous factors in influencing decision formation and how evidence is accumulated on multiple levels from the abstract, supramodal level to the effector-specific.

Variations in the quality of sensory evidence can originate not only from the physical environment but also from within the brain itself (Parker and Newsome, 1998; Sadaghiani et al., 2010). A key challenge is to disentangle these sources of variability and elucidate the nature of their influence on the decision making process (Hesselmann et al., 2010; de Lange et al., 2013). In addition to its strong sensitivity to the quality of the physical evidence, we found that the buildup rate of the CPP was subject to substantial within-coherence variability that determined the timing of decision reports on a trial-to-trial basis. The buildup rate of the CPP itself bore an inverse relationship with the level of $\alpha$-band 
activity immediately before evidence onset, a relationship that cannot be attributed to any exogenous factors because visual stimulation was held stationary during the intertarget interval. Whereas many previous studies have reported associations between the level of prestimulus activity in the $\alpha$ band and the timing or accuracy of behavioral performance (e.g., van Dijk et al., 2008; Kelly et al., 2009; O'Connell et al., 2009; Bengson et al., 2012), the present finding goes further by establishing the specific aspect of information processing that is augmented by pretarget attentional state to bring about these behavioral effects. It remains to be determined whether the attentional fluctuations indexed in pretarget $\alpha$ impact on accumulation rate directly or indirectly by influencing the initial encoding of sensory evidence (Brunton et al., 2013) or its weighting before integration (Wyart et al., 2012).

Unlike any other integrate-to-threshold signal observed in monkeys or humans, the CPP is fully abstracted from sensory and motor requirements (O'Connell et al., 2012). Although empirical observations of decision variable signals in the monkey brain have been limited to effector-selective regions, it is increasingly acknowledged that the full extent of behavioral flexibility inherent in human decision making cannot be achieved by a purely intentional framework where sensory evidence is directly incorporated into a motor plan (Heekeren et al., 2008; Bennur and Gold, 2011; Freedman and Assad, 2011; Wyart et al., 2012). Rather, it requires a hierarchical architecture within which evidence can be accrued at multiple levels of abstraction (Cisek, 2012; Dehaene and Sigman, 2012). Here we offer new insights by showing that the brain's representation of accrued evidence at the abstract, supramodal level is as closely tied to the quality of sensory evidence and the timing of decision reports as effectorselective signals have been shown to be in monkeys. Further, by tracking an effector-selective decision variable signal with the same temporal resolution in parallel, we have been able to establish that the buildup of accrued evidence at the abstract level significantly leads the buildup at the motor preparatory level, implying that the supramodal decision variable may intermediate between sensory encoding and motor planning. Despite the temporal lag, the two signals exhibit a simultaneous boundary crossing effect at commitment. This observation gives further insight into the form of the function relating abstract to premotor decision signals; in particular, it places the constraint that the latter cannot be the integral of the former because an equivalent boundary-crossing effect across coherences could not be observed for both signals in that case. At the same time, the substantial buildup in LRP before the CPP reaching its threshold level rules out a strictly serial interpretation of decision making (Sternberg, 1969) but rather supports the notion that sensory evidence flows continuously to the human motor system throughout decision formation (Selen et al., 2012).

The imperative to link human ERP components to the information processing stages intervening between sensation and response has a decades-long history. Indeed, the centroparietal "P300" or "P3b" component, which we have pointed out bears a strong resemblance to the CPP (O'Connell et al., 2012), was linked to decision making very soon after its discovery in 1965: it is evoked exclusively by task-relevant events requiring decisions (Sutton et al., 1965; Hillyard et al., 1971; Rohrbaugh et al., 1974); it discriminates hits from misses in a signal detection framework (Hillyard et al., 1971); and its latency varies closely with RT under conditions where accuracy is emphasized (Kutas et al., 1977) as well as with factors affecting the duration of stimulus evaluation, such as visual "noise" (McCarthy and Donchin, 1981). However, over the ensuing years, a consensus regarding the precise role played by the P300 in decision making has failed to emerge (Donchin and Coles, 1988; Kok, 2001; Nieuwenhuis et al., 2005; Verleger et al., 2005; Polich, 2007). This is in large part the result of the problem of global signal summation in typical discrete ERP paradigms (Magliero et al., 1984), in which functionally discrete signals overlap and therefore cannot be readily disentangled. In our current and previous study (O'Connell et al., 2012), the simple approach of eliminating sudden intensity transients at target onset has provided an unprecedented, unobscured view of the centroparietal decision signal and has enabled a concrete link to be made with the theoretical decision variables that lie at the center of well-established, mechanistic explanatory models. More generally, the current findings call for a novel view of the P300 component as a dynamically evolving decision process rather than as the culmination of a unitary event and highlight the prospect that a reevaluation of past P300 findings in this new light may inform models of human decision making as well as guide the next empirical steps.

\section{References}

Baker KS, Piriyapunyaporn T, Cunnington R (2012) Neural activity in readiness for incidental and explicitly timed actions. Neuropsychologia 50 : 715-722. CrossRef Medline

Bengson JJ, Mangun GR, Mazaheri A (2012) The neural markers of an imminent failure of response inhibition. Neuroimage 59:1534-1539. CrossRef Medline

Bennur S, Gold JI (2011) Distinct representations of a perceptual decision and the associated oculomotor plan in the monkey lateral intraparietal area. J Neurosci 31:913-921. CrossRef Medline

Bode S, Sewell DK, Lilburn S, Forte JD, Smith PL, Stahl J (2012) Predicting perceptual decision biases from early brain activity. J Neurosci 32:12488 12498. CrossRef Medline

Britten KH, Shadlen MN, Newsome WT, Movshon JA (1992) The analysis of visual motion: a comparison of neuronal and psychophysical performance. J Neurosci 12:4745-4765. Medline

Brunia CH, van Boxtel GJ (2001) Wait and see. Int J Psychophysiol 43:5975. CrossRef Medline

Brunton BW, Botvinick MM, Brody CD (2013) Rats and humans can optimally accumulate evidence for decision-making. Science 340:95-98. CrossRef Medline

Cisek P (2012) Making decisions through a distributed consensus. Curr Opin Neurobiol 22:927-936. CrossRef Medline

Dehaene S, Sigman M (2012) From a single decision to a multi-step algorithm. Curr Opin Neurobiol 22:937-945. CrossRef Medline

de Jong R, Wierda M, Mulder G, Mulder LJ (1988) Use of partial stimulus information in response processing. J Exp Psychol Hum Percept Perform 14:682-692. CrossRef Medline

de Lange FP, Jensen O, Dehaene S (2010) Accumulation of evidence during sequential decision making: the importance of top-down factors. J Neurosci 30:731-738. CrossRef Medline

de Lange FP, Rahnev DA, Donner TH, Lau H (2013) Prestimulus oscillatory activity over motor cortex reflects perceptual expectations. J Neurosci 33:1400-1410. CrossRef Medline

Delorme A, Makeig S (2004) EEGLAB: an open source toolbox for analysis of single-trial EEG dynamics including independent component analysis. J Neurosci Methods 134:9-21. CrossRef Medline

Donchin E, Coles MGH (1988) Is the P300 component a manifestation of context updating? Brain Behav Sci 11:357-374. CrossRef

Donner TH, Siegel M, Fries P, Engel AK (2009) Buildup of choicepredictive activity in human motor cortex during perceptual decision making. Curr Biol 19:1581-1585. CrossRef Medline

Eimer M (1998) The lateralized readiness potential as an on-line measure of central response activation processes. Behav Res Methods Instruments Computers 30:146-156. CrossRef

Foxe JJ, Simpson GV, Ahlfors SP (1998) Parieto-occipital approximately 10 $\mathrm{Hz}$ activity reflects anticipatory state of visual attention mechanisms. Neuroreport 9:3929-3933. CrossRef Medline

Freedman DJ, Assad JA (2011) A proposed common neural mechanism for 
categorization and perceptual decisions. Nat Neurosci 14:143-146. CrossRef Medline

Gold JI, Shadlen MN (2007) The neural basis of decision making. Annu Rev Neurosci 30:535-574. CrossRef Medline

Gratton G, Coles MG, Sirevaag EJ, Eriksen CW, Donchin E (1988) Pre- and post-stimulus activation of response channels: a psychophysiological analysis. J Exp Psychol Hum Percept Perform 14:331-344. CrossRef Medline

Heekeren HR, Marrett S, Ungerleider LG (2008) The neural systems that mediate human perceptual decision making. Nat Rev Neurosci 9:467479. CrossRef Medline

Hesselmann G, Sadaghiani S, Friston KJ, Kleinschmidt A (2010) Predictive coding or evidence accumulation? False inference and neuronal fluctuations. PLoS One 5:e9926. CrossRef Medline

Hillyard SA, Squires KC, Bauer JW, Lindsay PH (1971) Evoked potential correlates of auditory signal detection. Science 172:1357-1360. CrossRef Medline

Kayser J, Tenke CE (2006) Principal components analysis of Laplacian waveforms as a generic method for identifying ERP generator patterns: I. Evaluation with auditory oddball tasks. Clin Neurophysiol 117:348-368. CrossRef Medline

Kelly SP, Lalor EC, Reilly RB, Foxe JJ (2006) Increases in $\alpha$ oscillatory power reflect an active retinotopic mechanism for distracter suppression during sustained visuospatial attention. J Neurophysiol 95:3844-3851. CrossRef Medline

Kelly SP, Gomez-Ramirez M, Foxe JJ (2009) The strength of anticipatory spatial biasing predicts target discrimination at attended locations: a highdensity EEG study. Eur J Neurosci 30:2224-2234. CrossRef Medline

Kim JN, Shadlen MN (1999) Neural correlates of a decision in the dorsolateral prefrontal cortex of the macaque. Nat Neurosci 2:176-185. CrossRef Medline

Kok A (2001) On the utility of P3 amplitude as a measure of processing capacity. Psychophysiology 38:557-577. CrossRef Medline

Kutas M, McCarthy G, Donchin E (1977) Augmenting mental chronometry: the P300 as a measure of stimulus evaluation time. Science 197:792795. CrossRef Medline

Magliero A, Bashore TR, Coles MG, Donchin E (1984) On the dependence of P300 latency on stimulus evaluation processes. Psychophysiology 21: 171-186. CrossRef Medline

McCarthy G, Donchin E (1981) A metric for thought: a comparison of P300 latency and reaction time. Science 211:77-80. CrossRef Medline

Murphy PR, Robertson IH, Allen D, Hester R, O'Connell RG (2012) An electrophysiological signal that precisely tracks the emergence of error awareness. Front Hum Neurosci 6:epub. CrossRef Medline

Newsome WT, Britten KH, Movshon JA (1989) Neuronal correlates of a perceptual decision. Nature 341:52-54. CrossRef Medline

Nieuwenhuis S, Aston-Jones G, Cohen JD (2005) Decision making, the P3, and the locus coeruleus-norepinephrine system. Psychol Bull 131:510532. CrossRef Medline

O'Connell RG, Dockree PM, Robertson IH, Bellgrove MA, Foxe JJ, Kelly SP (2009) Uncovering the neural signature of lapsing attention: electrophysiological signals predict errors up to $20 \mathrm{~s}$ before they occur. J Neurosci 29:8604-8611. CrossRef Medline

O'Connell RG, Dockree PM, Kelly SP (2012) A supramodal accumulationto-bound signal that determines perceptual decisions in humans. Nat Neurosci 15:1729-1735. CrossRef Medline
Parker AJ, Newsome WT (1998) Sense and the single neuron: probing the physiology of perception. Annu Rev Neurosci 21:227-277. CrossRef Medline

Parra LC, Spence CD, Gerson AD, Sajda P (2005) Recipes for the linear analysis of EEG. Neuroimage 28:236-241. CrossRef Medline

Philiastides MG, Ratcliff R, Sajda P (2006) Neural representation of task difficulty and decision making during perceptual categorization: a timing diagram. J Neurosci 26:8965-8975. CrossRef Medline

Polich J (2007) Updating P300: an integrative theory of P3a and P3b. Clin Neurophysiol 118:2128-2148. CrossRef Medline

Ratcliff R, McKoon G (2008) The diffusion decision model: theory and data for two-choice decision tasks. Neural Comput 20:873-922. CrossRef Medline

Ratcliff R, Philiastides MG, Sajda P (2009) Quality of evidence for perceptual decision making is indexed by trial-to-trial variability of the EEG. Proc Natl Acad Sci U S A 106:6539-6544. CrossRef Medline

Rohrbaugh JW, Donchin E, Eriksen CW (1974) Decision making and the P300 component of the cortical evoked response. Percept Psychophys 15:368-374. CrossRef

Sadaghiani S, Hesselmann G, Friston KJ, Kleinschmidt A (2010) The relation of ongoing brain activity, evoked neural response, and cognition. Front Syst Neurosci 4:20. CrossRef Medline

Selen LP, Shadlen MN, Wolpert DM (2012) Deliberation in the motor system: reflex gains track evolving evidence leading to a decision. J Neurosci 32:2276-2286. CrossRef Medline

Shadlen MN, Newsome WT (2001) Neural basis of a perceptual decision in the parietal cortex (area LIP) of the rhesus monkey. J Neurophysiol 86: 1916-1936. Medline

Smith PL, Ratcliff R (2004) Psychology and neurobiology of simple decisions. Trends Neurosci 27:161-168. CrossRef Medline

Smith PL, Vickers D (1988) The accumulator model of two-choice discrimination. J Math Psychol 32:135-168. CrossRef

Sternberg S (1969) The discovery of processing stages: extensions of Donder's method. Acta Psychologica 30:276-315.

Sutton S, Braren M, Zubin J, John ER (1965) Evoked potential correlates of stimulus uncertainty. Science 150:1187-1188. CrossRef Medline

Thut G, Nietzel A, Brandt SA, Pascual-Leone A (2006) $\alpha$-Band electroencephalographic activity over occipital cortex indexes visuospatial attention bias and predicts visual target detection. J Neurosci 26:9494-9502. CrossRef Medline

van Dijk H, Schoffelen JM, Oostenveld R, Jensen O (2008) Prestimulus oscillatory activity in the $\alpha$ band predicts visual discrimination ability. J Neurosci 28:1816-1823. CrossRef Medline

van Vugt MK, Simen P, Nystrom LE, Holmes P, Cohen JD (2012) EEG oscillations reveal neural correlates of evidence accumulation. Front Neurosci 6:epub. CrossRef Medline

Vandekerckhove J, Tuerlinckx F (2008) Diffusion model analysis with MATLAB: a DMAT primer. Behav Res Methods 40:61-72. CrossRef Medline

Verleger R, Jaskowski P, Wascher E (2005) Evidence for an integrative role of P3b in linking reaction to perception. J Psychophysiol 19:165-181. CrossRef

Wyart V, de Gardelle V, Scholl J, Summerfield C (2012) Rhythmic fluctuations in evidence accumulation during decision making in the human brain. Neuron 76:847-858. CrossRef Medline 